\title{
Der Autor als Fragment
}

\author{
Romantische Herausgeberschaft (Hardenberg, Schlegel, Tieck)
}

\author{
Erika Thomalla
}

Autoren machen keine Werke, nicht einmal ihre eigenen. Zu Werken werden Texte erst, indem sie ausgewählt und angeordnet, gekürzt, umgeschrieben oder ergänzt, gerahmt, präsentiert und in Umlauf gebracht werden. Werke sind immer schon die Produkte arbeitsteiliger Kollektive, auch und gerade dann, wenn viele der beteiligten Akteure am Ende nicht auf dem Buchcover erscheinen. ${ }^{1}$ Autorschaft im Singular verdankt sich stets einer Vielzahl an Kollaborationen. Dabei handelt es sich nicht notwendig um emphatische Formen der Zusammenarbeit, die auf Konsens und gemeinsamen Interessen beruhen. Werke und ihre Autoren sind in der Regel das Ergebnis kollektiver Praktiken, die zerstreut und vermittelt, nicht immer abgestimmt und zum Teil nicht einmal gewollt sind. Herausgeber wissen das. Seit Autoren sich als Schöpfer ihrer Werke begreifen, erliegen Herausgeber dem Phantasma, an die Stelle von Autoren treten und deren Tätigkeit noch vollendeter ausüben zu können als sie selbst. ${ }^{2}$ Erst durch editoriale Verfahren nimmt Geschriebenes jene scheinbar authentische und überzeitliche Form an, die es als tradierungswürdigen, kanonischen oder klassischen Text lesbar macht. Herausgeber machen Texte zuallererst zu Werken eines Autors oder eines Autorenkollektivs. ${ }^{3}$

1 Vgl. Stephan Porombka, „Literaturbetriebskunde. Zur ,genetischen Kritik kollektiver Kreativität“, in: Stephan Porombka, Wolfgang Schneider und Volker Wortmann (Hrsg.), Kollektive Kreativität, Tübingen: Narr 2006, S. 71-86.

2 Vgl. Wolf Kittler, „Literatur, Edition und Reprographie“, in: Deutsche Vierteljahrsschrift für Literaturwissenschaft und Geistesgeschichte 65 (1991), H. 2, S. 205-235, hier bes. S. 209.

3 Dabei kann es sich selbstverständlich auch um Selbstherausgeberschaften handeln. Der Ansatz, Autorschaft als das Produkt editorialer Praktiken zu untersuchen, schließt an die Thesen Uwe Wirths an, der sich in seiner Studie über die "Geburt des Autors aus dem Geist der Herausgeberfiktion" allerdings auf den Sonderfall des fiktiven Herausgebers beschränkt und deshalb die ökonomischen, sozialen, medialen und politischen Bedingungen von Herausgeberschaften nicht berücksichtigt. Diese Kontexte sind für die Entstehung neuer Autorschafts- und Werkkonzepte im 18. Jahrhundert jedoch maßgeblich. Vgl. Uwe Wirth, Die Geburt des Autors aus dem Geist der Herausgeberfiktion. Editoriale Rahmung im Roman um 1800: Wieland, Goethe, Brentano, Jean Paul und E.T.A. Hoffmann, München: Fink 20o8, bes. S. 13. Für eine umfassende Geschichte der modernen Herausgeberschaft siehe Erika Thomalla, Anwälte des Autors. Zur Geschichte der Herausgeberschaft im 18. und 19. Jahrhundert, Göttingen: Wallstein 2020.

(C) ERIKA THOMALLA, 2022 | DOI:10.30965/9783846766569_006

This is an open access chapter distributed under the terms of the CC BY-NC-ND 4.q license nomal la - 9783846766569 
Ein Autor, für den das in besonderer Weise gilt, ist Friedrich von Hardenberg. Als Hardenberg am 25. März 1801 im Alter von 28 Jahren starb, hatte er nur wenig veröffentlicht. Auf sein Debüt, das Gedicht Klagen eines Jünglings im Teutschen Merkur (1791), folgten lediglich drei weitere Publikationen: Unter dem Pseudonym Novalis erschienen 1798 die Fragment-Sammlungen Blumen / Glaube und Liebe in den Jahrbüchern der preußischen Monarchie sowie die Blüthenstaub-Fragmente im Athenäum. Zwei Jahre später wurden die Hymnen an die Nacht ebenfalls im Athenäum veröffentlicht. Seinen eigentlichen Ruhm als Autor erlangte Novalis erst posthum. Dazu trugen ganz wesentlich seine Freunde Ludwig Tieck und Friedrich Schlegel bei, die bereits 1802 das Romanfragment Heinrich von Ofterdingen sowie eine zweibändige Ausgabe mit dem Titel Novalis Schriften herausgaben. Während der Roman sich in der Einzelausgabe nur sehr "langsam“ verkaufte, wurde die Werkedition zum Publikumserfolg - sie erreichte bis 1837 fünf Auflagen. ${ }^{4}$

Ein Hauptgrund für diese Popularität war die Editionsstrategie Schlegels und Tiecks. Sie präsentierten Novalis als einen Dichter, der in jeder Hinsicht Fragment geblieben war und der vorgeblich nichts als Fragmente hinterlassen hatte. Damit bahnten sie den Weg für vielfältige Rezeptions- und Aneignungsmöglichkeiten. Weil die romantische „Leitmetapher" des Fragments "semantisch offen“ ist, ihr „Korrelat, das Ganze, in der Schwebe läßt“ und zur interpretierenden Vervollständigung auffordert, ${ }^{5}$ konnte Novalis' Werk im 19. Jahrhundert als Relikt einer ehemaligen wie auch als Anzeichen einer noch nicht zur Vollendung gekommenen Ganzheit gedeutet werden. Seine Schriften waren gleichermaßen als Überrest oder Spur der verstorbenen Person, als unvollendeter Auftakt eines literarischen Epochenwechsels oder als Initiation einer politischen, ästhetischen oder religiösen Mission lesbar. Die fragmentarische Gestalt des Werks stattete es mit einem erhöhten Maß an Polysemie aus, die den Liebhabern klassischer Texte entgegenkam. Der „herrliche Novalis", so sahen es viele seiner Leser, hatte "göttliche[] Ahndungen“6 oder "heilige, unerschöpflich sinnreiche Zeichen“ hinterlassen, ${ }^{7}$ die der Deutung, Erläuterung und Vollendung harrten.

4 Doris Reimer, Passion und Kalkül. Der Verleger Georg Andreas Reimer (1776-1842), Berlin, New York: de Gruyter 1999, S. 305.

5 Eberhard Ostermann, „Der Begriff des Fragments als Leitmetapher der ästhetischen Moderne“, in: Athenäum. Jahrbuch für Romantik 1 (1991), S. 189-205, hier S. 190.

6 Brief von Karl August Varnhagen von Ense an Elise Müller vom 28.07.1805, in: Karl August Varnhagen von Ense, Aus dem Nachlaß Varnhagen's von Ense. Briefe von der Universität in die Heimath, hrsg. von Ludmilla Assing, Brockhaus: Leipzig 1874, S. 224 und S. 230.

7 Adam Müller, „Vorlesungen über die deutsche Wissenschaft und Literatur, gehalten zu Dresden im Winter 1806“, in: ders., Kritische, asthetische und philosophische Schriften, Bd. I, 


\section{Verrätselung. Informationsdefizite schaffen}

Schlegel und Tieck zeichneten die Linien, die Hardenbergs Rezeption in der Literaturgeschichte bestimmt haben, in den Schriften vor. Sowohl programmatisch - in den Peri- und Epitexten - als auch durch ihre editionspraktische Tätigkeit prägten sie ein spezifisches Bild des Autors. Dabei schufen sie zunächst einen grundlegenden Informationsmangel. Über die Person und das Leben des Autors ließen sie die Leser im Unklaren. In der Vorrede zur Erstausgabe greift Tieck auf einen beliebten Topos zurück, indem er die Leser als „Freunde“ des Verfassers adressiert, die dessen „innere Geschichte“ sowie die „Andeutungen“ seines „veränderten Gemüths“ aus den hinterlassenen „Fragmenten" auch ohne biographische Kontextinformationen erahnen können müssten. Die von den Herausgebern veröffentlichten Bruchstücke, so die Behauptung, „drücken [...] das Gemüth des Verfassers“ selbst „vollkommen“ aus. ${ }^{8}$ Tieck erklärt die Ausklammerung ,äußerer' Daten - Lebensstationen, Begebenheiten oder Personen - geradezu zur Möglichkeitsbedingung eines tieferen Verständnisses. Das Werk offenbart das Leben und den Charakter der Person, nicht umgekehrt. ${ }^{9}$

Mit der Auffassung, dass der Charakter einer Person so komplex und vielseitig sei, dass er niemals im Ganzen, sondern nur durch einzelne Fragmente oder Bruchstücke erfasst werden könne, bewegten sich Schlegel und Tieck auf der Höhe der Charaktertheorien ihrer Zeit. ${ }^{10}$ Tagebücher und Biographien, die auf das Fragmentarische ihrer Darstellung hinwiesen, waren bereits seit mehreren Jahren in Mode: Vor allem in den 8oer und goer Jahren des 18. Jahrhunderts erschienen zahlreiche Publikationen mit Titeln wie Fragmente zu dem Leben des Grafen von Herzberg (1796), Fragmente zur Biographie des verstorbenen geheimen Raths Bode (1795), Fragmente zur Schilderung des Geistes,

kritische Ausgabe, hrsg. von Walter Schroeder und Werner Siebert, Neuwied, Berlin: Luchterhand 1967, S. 13-137, hier S. 56 .

8 Ludwig Tieck, „Vorrede“, in: Friedrich von Hardenberg, Novalis Schriften, hrsg. von Friedrich Schlegel und Ludwig Tieck, Erster Theil, Berlin: Buchhandlung der Realschule 1802, S. I-XII, hier S. If.

9 Vgl. zu dieser Konzeptualisierung des Leben-Werk-Zusammenhangs bei Tieck Roger Paulin, „Künstlerbiographie, Hagiographie und persönliches Schicksal. Zu Tiecks Kleistbild und seinem Hintergrund“, in: Sabine Doering, Waltraud Maierhofer und Peter Philipp Riedl (Hrsg.), Resonanzen. Festschrift für Hans Joachim Kreutzer zum 65. Geburtstag, Würzburg: Königshausen \& Neumann 200o, S. 329-341, hier S. 333ff.

10 Vgl. Erika Thomalla, „Totalcharakter. Die Entzifferung des Subjekts im 18. Jahrhundert“, in: Michael Hohlstein, Rudolf Schlögl und Isabelle Schürch (Hrsg.), Der Mensch in Gesellschaft. Zur Vorgeschichte des modernen Subjekts in der Frühen Neuzeit, Paderborn u.a.: Schöningh 2019, S. $265^{-287}$, bes. S. $28{ }_{3} \mathrm{ff}$. 
des Charakters, und der Regierung Friedrich des Zweiten (1798) oder Fragmente über Italien aus dem Tagebuche eines jungen Deutschen (1798). Tiecks Vorwort schließt implizit an diese Mode an, treibt sie aber auf die Spitze, indem es der traditionellen Form der Lebensbeschreibung gar keine Explikationsleistung mehr zutraut. Nur noch das fragmentarische Werk selbst kann Zugang zum Charakter bieten.

Die Ansprüche an eine gelungene Rezeption sind entsprechend hoch. Die Leser sollen die Lebensgeschichte eines Autors, den viele von ihnen bloß unter seinem Pseudonym kennen, aus Textfragmenten erschließen, die keine nachvollziehbare Anordnung aufweisen: Die Schriften sind nicht chronologisch oder generisch geordnet, sondern sie beginnen mit dem nachgelassenen Romanfragment Heinrich von Ofterdingen und enden mit Fragmenten vermischten Inhalts, einer von Tieck und Schlegel verantworteten Zusammenstellung von Textauszügen, die Hardenberg mehrere Jahre vor seinem Tod schrieb und bloß in Teilen veröffentlichte. Die Herausforderung, in diesen Textfragmenten eine "Geschichte“ zu erkennen, bleibt dem Publikum überlassen. Doch damit ist erst ein Teil der Lektüreanweisungen benannt: Das Zentrum der Schriften bildet Tieck zufolge der „Geist“ des Verfassers, der die „verschiedensten Gedanken“ verbinde und zusammenhalte. Ein „näher befreundetes Gemüth“, so die zuversichtliche Vermutung des Herausgebers, werde die Gemeinsamkeit der einzelnen Bruchstücke mühelos erkennen. Die Dynamik einer individuellen Entwicklungsgeschichte soll also mit der Totalitäts- und Ganzheitskonzeption eines gleichbleibenden Charakters oder Geists kombiniert werden. Die Werkfragmente und die Person werden dabei nach dem Modell eines hermeneutischen Zirkels in Beziehung gesetzt:Während die vereinzelten "Gedanken“ den alles umfassenden „Geist" enthalten, macht dieser Geist „wie aus einem Mittelpunkte alle übrigen [Gedanken] verständlich“..11

Novalis „vollkommen“ zu verstehen, heißt also offenbar, unterschiedliche Äußerungen, Textgattungen und gedankliche Wandlungen in seinen Texten mit einer ideellen Einheit in Einklang zu bringen. Die methodische Versiertheit, die den Lesern der Schriften damit abverlangt wird, lässt darauf schließen, dass es sich bei den von Tieck adressierten „Freunden“ um Freunde oder Liebende des Worts - kurz: um Philologen - handelt, oder zumindest um Leser, die mit philologischen und hermeneutischen Lektüreverfahren vertraut sind. Die Wahl des Verlags der Berliner Realschulbuchhandlung ${ }^{12}$ und

11 Tieck, „Vorrede“ (Anm. 8), S. XI.

12 Ursprünglich verhandelten Tieck und die Brüder Schlegel mit dem Verleger Johann Friedrich Unger, da Novalis den Roman Heinrich von Ofterdingen beim selben Verlag und in derselben „Gestalt“ veröffentlichen wollte, in der auch Goethes Wilhelm Meister 
die geringe Erstauflagenhöhe von 5oo Exemplaren sprechen ebenfalls für die Vermutung, dass die Ausgabe sich eher an ein literaturtheoretisch geschultes Publikum statt an den populären Buchmarkt richtete. Der seit 180o von Georg Andreas Reimer geführte Realschulbuchhandlungsverlag galt nicht nur wegen seiner Nähe zu den Autoren der Frühromantik als fortschrittlich und modern, sondern er suchte vor allem gezielt den Anschluss an zeitgenössische wissenschaftliche Entwicklungen, die er zu einem allgemeinen Bildungsgut machen wollte. ${ }^{13}$ Einer der ersten Autoren, die Reimer verlegte, war Friedrich Schleiermacher. In den darauffolgenden Jahren wurde der Verlag für viele deutsche Philologen - etwa für Karl Lachmann, die Brüder Grimm oder Georg Friedrich Benecke - zu einem Hauptpublikationsort.

Für die „Freunde“ dieses Verlagsprogramms sollte es also möglich sein, den Geist und die Entwicklungsgeschichte eines frühverstorbenen Autors in seinen Texten aufzufinden. Erst als die Novalis-Ausgabe 1815 ihre dritte Auflage erreichte, bereicherte Tieck sie durch eine Biographie, die auf einer Niederschrift von Novalis' Bruder Karl von Hardenberg beruhte. Die Auffassung, dass nicht der Lebenslauf das Werk erkläre, sondern dass "Geist“, „Wandel“ und „persönliche Gestalt" eines Dichters sich nur in der "wiederholten Lecture“ seiner Texte erschließen, behielt er allerdings bei. Tiecks Biographie ist kein Tatsachenbericht, sondern eine hagiographische, verklärende Darstellung. Sie folgt der Direktive, das Leben „als ein Gedicht [zu] lesen“, indem der fehlende „Zusammenhang“ zwischen den Einzelereignissen selbst „ergänzt" wird. ${ }^{14}$ Das "Gedicht", das Tieck vom Leben Hardenbergs entwirft, wirkte sich nachhaltig auf dessen Wahrnehmung in der literarischen Öffentlichkeit des 19. Jahrhunderts aus. ${ }^{15}$ Bereits rein äußerlich rückt Tieck seinen Freund in die Nähe

erschienen war. Weil Unger aber zögerte und das finanzielle Risiko offenbar nicht eingehen wollte, wandten sie sich zunächst an Friedrich Fromann und dann an Reimer. Vgl. dazu den Briefwechsel zwischen Tieck, August Wilhelm und Friedrich Schlegel zwischen dem 30.06.1801 und dem 04.04.1802, in: Friedrich von Hardenberg, Novalis Schriften, Historisch-kritische Ausgabe, Bd. 5, hrsg. von Hans-Joachim Mähl und Richard Samuel, Stuttgart: Kohlhammer 1988, S. 137-148.

13 Vgl. Reimer, Passion und Kalkül (Anm. 4), S. 61ff.

14 Ludwig Tieck, Kritische Schriften, zum erstenmale gesammelt und mit einer Vorrede hrsg. von demselben, Leipzig: Brockhaus 1848, S. 18 o.

15 Auch in seinen anderen Editionsprojekten, etwa den Ausgaben der Schriften Heinrich von Kleists oder Jakob Michael Reinhold Lenz', haben die Biographien eher einen paradigmatischen Charakter, die der exemplarischen Darstellung gescheiterter oder marginalisierter Künstlerexistenzen sowie deren Verortung innerhalb von literaturhistorischen Großzusammenhängen dient. Vgl. Paulin, „Künstlerbiographie“ (Anm. 9), S. 337ff;; Marek Zybura, Ludwig Tieck als Übersetzer und Herausgeber, Heidelberg: Winter 1994, S. $147 \mathrm{ff}$. 
eines Heiligen: Sein Auge war „hell“ und die „Farbe seines Gesichtes [...] fast durchsichtig“. Sein Gesichtsausdruck „kam sehr dem Evangelisten Johannes nahe, wie wir ihn auf der herrlichen großen Tafel von A. Dürer sehn“. ${ }^{16}$ Dieser sakralisierende Vergleich verbindet sich mit der Aufforderung des Herausgebers, die Schriften von Novalis als „Buch der Erweckung und Andacht“ zu lesen. ${ }^{17}$ Damit erhalten die Fragmente den Charakter einer Offenbarungsschrift mit prophetischem Potenzial.

Als ein Heiliger, der der Welt schon bei Lebzeiten entrückt war, erscheint Novalis auch in Tiecks Beschreibung seiner Persönlichkeit. Sein ganzes "Wesen und alle seine Vorstellungen“ waren von einer „innigen Liebe“ und einer „frommen Todessehnsucht" bestimmt. Ein „einziger großer LebensMoment“, der Tod seiner Verlobten Sophie von Kühn im Alter von 15 Jahren, sei der Schlüssel zum „Wesen seiner Poesie und Anschauung“ ${ }^{18}$ Mit dieser Begründung verleiht Tieck den Schriften nachträglich jenes geistige Zentrum, dessen Bestimmung er 1802 noch als Suchauftrag an die Leser gerichtet hatte. Dass dabei wesentliche Momente von Hardenbergs Leben marginalisiert werden - etwa seine rege Berufstätigkeit, seine Vielleserei oder seine erneute Verlobung mit Julie von Charpentier 1798 -, ist von der Forschung vielfach kritisiert worden, ${ }^{19}$ erscheint in der Logik Tiecks aber durchaus konsequent. Sein Verfahren entspricht zudem einer Tendenz in der zeitgenössischen Biographik, weniger einzelne Daten und Ereignisse zu dokumentieren, sondern Synthesen zu bilden, um Aussagen über das "ganze“ Wesen einer Person treffen zu können. ${ }^{20}$ Poetisierungen und stilisierende Verklärungen sind in dieser biographischen Tradition durchaus zulässig.

Wie stark Tieck damit dem Geschmack des Publikums entsprach, lässt sich auch ex negativo zeigen: Als der Arzt und Literat Justinus Kerner, der seinerseits eine Vorliebe für Hagiographien und Erbauungsbücher hatte, ${ }^{21} \mathrm{im} \mathrm{Jahr}$

16 Ludwig Tieck, „Vorrede zur dritten Auflage“, in: Friedrich von Hardenberg, Novalis Schriften, hrsg. von Ludwig Tieck und Friedrich Schlegel, Erster Theil, 3. Aufl., Berlin: Verlag der Realschulbuchhandlung 1815, S. XI-XXXVII, hier S. XXXIII.

17 Tieck, „Vorrede“ (Anm. 8), S. XXII.

18 Tieck, „Vorrede zur dritten Auflage“ (Anm. 16), S. XXI und S. XXXVII.

19 Vgl. Herbert Uerlings, „Tiecks Novalis-Edition“, in: Walter Schmitz (Hrsg.), Ludwig Tieck. Literaturprogramm und Lebensinszenierung im Kontext seiner Zeit, Tübingen: Niemeyer 1996, S. 135-159, hier S. $143^{\mathrm{ff}}$.

20 Hans-Martin Kruckis, „Ein potenziertes Abbild der Menschheit“. Biographischer Diskurs und Etablierung der Neugermanistik in der Goethe-Biographik bis Gundolf, Heidelberg: Winter 1995, S. $45 \mathrm{ff}$.

21 Kerner machte seine Patientin Friederike Hauffe, die in den letzten Lebensjahren bei ihm wohnte, als „Seherin von Prevorst“ bekannt. Das Leben Hauffes beschreibt Kerner als Parallelgeschichte von körperlichem Verfall und spiritueller Erweckung. Vgl. Justinus 
1810 einen Hardenberg-Nachruf in Friedrich von Schlichtegrolls Nekrolog der Teutschen für das 19. Jahrhundert (1805) gelesen hatte, in dem der Verstorbene in erster Linie als "Chursächsischer Salinenassessor" und „designirter Amshauptmann in Thüringen" gewürdigt wurde, ${ }^{22}$ erschien ihm diese Vorstellung schlicht „entsetzlich“. Die Zweitverlobung mit Julie von Charpentier hielt er für höchst unpoetisch. Lediglich am „schön[en]“ Tod des Dichters hatte er nichts auszusetzen. ${ }^{23}$ Die Entscheidung der Herausgeber Tieck und Schlegel, die dritte Auflage der Schriften durch eine Biographie zu erweitern, kann also auch als Versuch gedeutet werden, der nüchtern-prosaischen Lebensbeschreibung des vielgelesenen Nekrologs der Teutschen wieder ein anderes, ,poetischeres' Novalis-Bild entgegenzusetzen.

\section{Winke und Bruchstücke. Die Deutungen kühn kombinierender Geister}

Tiecks und Schlegels Bemühen, Novalis' „Geist“ zu „charakterisieren“, beschränkte sich nicht bloß auf die Vorreden. Die Frage, welche Texte und Darstellungsformen den "Charakter des Schriftstellers" am angemessensten repräsentierten, ${ }^{24}$ prägte auch den praktischen Umgang mit dessen Nachlass. Einen ersten Streitpunkt bildete das Romanfragment Heinrich von Afterdingen, dessen Titel die Herausgeber eigenmächtig in Heinrich von Ofterdingen umschrieben. ${ }^{25}$ Während Tieck sich von August Wilhelm Schlegel und

Kerner, Die Seherin von Prevorst. Eröffnungen über das innere Leben des Menschen und über das Hereinragen einer Geisterwelt in die unsere (1829), 2. verm. und verb. Aufl., Stuttgart, Tübingen: Cotta 1832 .

22 Der Nekrolog stammt von Coelestin August Just, „Friedrich von Hardenberg. Chursächsischer Salinenassessor und designirter Amtshauptmann in Thüringen“, in: Nekrolog der Teutschen für das 19. Jahrhundert, hrsg. von Friedrich Schlichtegroll, Bd. 4, Gotha: Justus Perthes 1805, S. 187-261.

23 Brief von Justinus Kerner an Ludwig Uhland vom 25.01.1810, in: Justinus Kerners Briefwechsel mit seinen Freunden, hrsg. von Theobald Kerner, Bd. 1, Stuttgart, Leipzig: Deutsche Verlags-Anstalt 1897, S. 95 .

24 Brief von Friedrich Schlegel an Ludwig Tieck vom 05.11.1801, in: Hardenberg, Novalis Schriften, Bd. 5 (Anm. 12), S. 145 .

25 Obwohl diese Namensänderung seit langem bekannt war, wurde sie selbst in der historisch-kritischen Ausgabe nicht wieder rückgängig gemacht. Eine kritische Neuedition, die einen Paralleldruck von Faksimile und Transkription mit editorischem Bericht und Interpretation enthält, bietet erst die Arbeit von Alexander Knopf aus dem Jahr 2015. Vgl. Friedrich von Hardenberg, „Begeisterung der Sprache“. Heinrich von Afterdingen. Textkritische Edition und Interpretation, hrsg. von Alexander Knopf, Frankfurt/ Main: Stroemfeld 2015. 
Friedrich Schleiermacher davon überzeugen ließ, den Roman selbst zu vollenden, war Friedrich Schlegel über diese „sündlich[e]“ und „unverzeihlich[e]“ Absicht so „empört", dass die drei anderen schnell wieder von dem Unternehmen Abstand nahmen. ${ }^{26}$ Allerdings ist dieser vehemente Einspruch nicht auf generelle Vorbehalte Schlegels gegenüber Eingriffen in den Nachlass zurückzuführen. Vielmehr kann vermutet werden, dass es ihm um den Erhalt der fragmentarischen Form selbst ging. Seine Einwände richteten sich primär gegen die Ganzheitsästhetik, die hinter Tiecks, Schleiermachers und August Wilhelm Schlegels Idee stand. Deren „verkehrten Begriffen von Fertigseyn, Fortsetzen, Vollenden“ hielt er das Fragmentarische als „Triumph“ der ästhetischen Form entgegen. ${ }^{27}$ Aus diesem Grund hatte er zwar Schwierigkeiten mit der Praktik des Ergänzens, nicht aber mit der des Kürzens und Streichens. ${ }^{28}$

Gekürzt wurde bei der Zusammenstellung der Schriften reichlich. Der Eindruck, Novalis habe nichts als Fragmente hinterlassen, ist das Ergebnis gezielter editorischer Bearbeitungen. Die Fragmente vermischten Inhalts, die am Ende des zweiten Bands der Schriften stehen, sind eine Mischung aus authentischen Fragmenten, die aus Glauben und Liebe oder aus dem Blüthenstaub stammen, und Auszügen aus Aufsätzen, Notizen oder Exzerpten, die Schlegel und Tieck zuallererst zu Fragmenten machten. Sie wählten einzelne Passagen aus Hardenbergs Aufzeichnungen aus, schrieben sie um, kürzten und kombinierten nach eigenem Ermessen. Schlegel kam dabei die Aufgabe der Auswahl zu, während Tieck die Anordnung übernahm. ${ }^{29}$

26 Brief von Friedrich Schlegel an Friedrich Schleiermacher um den 17.04.1801, in: Hardenberg, Novalis Schriften, Bd. 5 (Anm. 12), S. 134.

27 Ebd.

28 Bereits die Blüthenstaub-Fragmente, die Novalis für das Athenäum ausgewählt hatte, wurden von Schlegel bearbeitet, zerteilt und neu zusammengestellt. Vgl. dazu Helmut Schanze, „Dualismus unsrer Symphilosophie‘. Zum Verhältnis Novalis - Friedrich Schlegel", in:Jahrbuch des freien deutschen Hochstifts (1966), S. 309-335.

29 Die Frage, wie groß Schlegels Anteil bei der Zusammenstellung der Fragmente war, ist umstritten. Offenbar standen ihm die Notizen nur wenige Wochen (Ende April bis Mitte Mai 1802) zur Verfügung, in denen er - wie der handschriftliche Nachlass im Archiv des Freien deutschen Hochstifts in Frankfurt am Main zeigt - Anstreichungen vornahm und einzelne Sätze oder Absätze auswählte. Zudem entwarf Schlegel den Plan einer Anordnung in sechs Abteilungen. Dass er auch auf Formulierungen Einfluss nahm, ist nicht bekannt. Sicher ist aber, dass er mit dem Endergebnis zufrieden war und in einer späteren Ausgabe lediglich die Anordnung noch einmal änderte. Wenn im Folgenden von Tieck und Schlegel als Redaktoren der Fragmente die Rede ist, so geschieht dies im Bewusstsein ihrer Aufgabenteilung. Vgl. Richard Samuel, „Vorrede zum 2. und 3. Bande“, in: Friedrich von Hardenberg, Novalis Schriften. Historisch-kritische Ausgabe, Bd. 2, hrsg. von Richard Samuel in Zusammenarbeit mit Hans-Joachim Mähl und Gerhard Schulz, Stuttgart: Kohlhammer 1964 S. V-VIII; ders., „Zur Geschichte des Nachlasses Friedrich 
Die Theorie für dieses editorische Programm hatte Schlegel bereits einige Jahre zuvor an einem anderen Beispiel erarbeitet. ${ }^{30}$ Auch Gotthold Ephraim Lessing war für Schlegel, wie er 1797 in einem Aufsatz erläuterte, ein fragmentarischer Autor: Alles, was Lessing hinterlassen habe, seien "Winke und Andeutungen“ oder „Bruchstücke von Bruchstücken“. Lessings vollkommenste Werke bildeten angeblich „nur eine Kette von witzigen Einfällen“. ${ }^{31}$ Diese unwahrscheinliche Behauptung begründet Schlegel, indem er einen Großteil von Lessings Schriften als unoriginell oder qualitativ minderwertig beurteilt. Die Dichtungen schließt er - mit Ausnahme des Dramas Nathan der Weise, bei dem es sich um einen philosophischen Text handle - aus dem Gesamtwerk aus, weil Lessing kein Poet im eigentlichen Sinn, sondern Philosoph und vor allem Kritiker gewesen sei. Von den übrigen Schriften werden nur diejenigen akzeptiert, die nach Schlegels Auffassung „die eigenen Gedanken Lessings" widerspiegeln. Als er 1804 Lessings Gedanken und Meinungen aus dessen Schriften edierte, ließ er deshalb alles weg, was er als "gleichgültig“, "störend“, veraltet oder fremd ansah. Im Ergebnis blieben „von einem großen Theil der Lessingschen Schriften nur Fragmente zurück“, von denen Schlegel behauptete, dass sie es "ganz ohne unser Zuthun geworden sind, oder vielmehr es gleich von Anfange an waren“. Die von Schlegel ausgewählten Textfragmente sollten demnach Lessings Gedanken in ihrer „ursprünglichen Gestalt“ darstellen. ${ }^{32}$

Die Pointe dieser editorialen Technik der Fragmentarisierung besteht darin, dass es sich vorgeblich nicht um eine Reduktion, sondern um eine Bereicherung handelt: Gerade die Zusammenstellung einzelner Versatzteile soll den "ganzen“ Lessing zur Anschauung bringen. Denn die Genialität Lessings sei nicht „im Einzelnen“, sondern in der "Gedankenreihe" aufzusuchen. Nicht der Inhalt, sondern die „Form“ der Fragmente sei entscheidend, und nur in ihrer Abfolge werde der "Witz“ eines „kühn combinirende[n] Geist[s]“ sichtbar. ${ }^{33}$ Dieser kombinatorische Genius scheintallerdings gerade nicht der des Autors, sondern der des Herausgebers zu sein, der ja allein für die Form und die Anordnung der

von Hardenbergs (Novalis)“, in: Jahrbuch der Deutschen Schillergesellschaft 2 (1958), S. $301-347$, bes. S. $313 \mathrm{ff}$.

30 Vgl. zum Folgenden auch Stefan Höhle, „Friedrich Schlegels Auseinandersetzung mit Lessing. Zum Problem des Verhältnisses zwischen Romantik und Aufklärung“, in: Weimarer Beiträge 23 (1977), H. 2, S. 122-135. hrsg. von Hans Eichner, Paderborn u.a.: Schöningh 1967, S. 10o-125, hier S. 112.

32 Friedrich Schlegel, Lessings Gedanken und Meinungen aus dessen Schriften zusammengestellt und erläutert von Friedrich Schlegel, Zweiter Theil, Leipzig: Juniuss 1804, S. 3. Ebd., Erster Theil, S. 8 und 18; ebd., Zweiter Theil, S. 16. 
Textausschnitte verantwortlich ist. Damit wird die Stoßrichtung von Schlegels Editionsprojekt deutlich: Lessings Gedanken bilden lediglich das „Organon einer noch zu vollendenden, zu bildenden, ja anzufangenden Litteratur“, deren eigentliche Begründung dem „combinatorischen Geist“ von Herausgeberautoren überlassen bleibt. ${ }^{34}$ Die "Charakterisierung“ des Aufklärungsschriftstellers mündet im Phantasma eines romantischen Selbstentwurfs. ${ }^{35}$

Dieser literaturgeschichtliche Brückenschlag bildet auch die Verbindung zwischen Schlegels Lessing-Ausgabe und den Novalis-Schriften Schlegels und Tiecks. Es ist kaum ein Zufall, dass Schlegel aus Lessings Werk einen einzigen Satz hervorhebt, der ihn "so wert" mache, dass man ihn allein dafür „ehren und lieben“ müsse, auch wenn er sonst „nichts bedeutendes" gesagt hätte. Es handelt sich - in der Auslegung Schlegels - um die Prophezeiung eines literarischen Neubeginns. Sie steht unter dem Titel „Etwas das Lessing gesagt hat" in der überarbeiteten Fassung von Schlegels Aufsatz Ueber Lessing (1801): „Es wird das neue Evangelium kommen.' - / So sagte Lessing Doch die blöde Rotte / Gewahrte nicht der aufgeschloßnen Pforte“. ${ }^{36}$ Was in Lessings Erziehung des Menschengeschlechts Teil einer humanistischen, aufklärerischen Utopie war, ${ }^{37}$ interpretiert Schlegel als ästhetische Utopie - als Voraussage einer „neue[n] Zeit“, in der "die Künste sich erneuen“ werden. ${ }^{38}$ Lessing erscheint damit als Prophet der romantischen Ära. Hardenberg wiederum wird von seinen verstorbenen Freunden als Evangelist dieser Offenbarung eingesetzt.

Die Gedankenfigur einer unmittelbaren Nachfolge Hardenbergs auf Lessing wurde von den romantischen Zeitgenossen im frühen 19. Jahrhundert bereitwillig aufgegriffen. Dies zeigt etwa eine Bemerkung Adam Müllers aus seinen Vorlesungen über die deutsche Literatur und Wissenschaft (1806). In jedem der „heiligen“ Fragmente von Novalis, so Müller, gebe es „ein unsichtbares Verlangen, die andern zu ergreifen und so das neue Evangelium, das

34 Ebd., Zweiter Theil, S. 3 und 11.

35 Dass Schlegel eine solche Fragmentarizität und Lückenhaftigkeit vor allem der modernen Kunst bescheinigt, die im Zuge eines heilsgeschichtlichen Prozesses wieder zu einer Einheit gelangen soll, betont Ethel Matala de Mazza, „Alle Protestanten sind zu betrachten als zukünftige Katholiken'. Friedrich Schlegels Konversionen“, in: Athenäum.Jahrbuch der Friedrich-Schlegel-Gesellschaft 18 (2008), S. 101-121.

36 Friedrich Schlegel, „Über Lessing“, in: August Wilhelm Schlegel und Friedrich Schlegel, Charakteristiken und Kritiken, Erster Band, Königsberg: Nicolovius 1801, S. 170-281, hier S. $222 f$.

37 Vgl. Gotthold Ephraim Lessing, „Die Erziehung des Menschengeschlechts“, in: ders., Werke, Bd. 8, in Zusammenarbeit mit Karl Eibl u.a. hrsg. von Herbert G. Göpfert, München: Hanser 1979, S. 489-510, hier S. 508. 
uns einst Lessing verhieß, zu bilden." Was Lessing verkündete, ,verlangte Novalis demnach zu erfüllen. Doch weil er diese Offenbarung nicht „deutlich“, sondern nur fragmentarisch ausdrücken konnte, war auch er auf Interpreten angewiesen, die seine „Ahndungen“ in Klartext übersetzten. ${ }^{39}$

Auch Novalis' „Evangelium“ ist daher in Teilen das Produkt kühn kombinierender Geister. Mehr noch: Schlegel und Tieck verleihen vielen von Hardenbergs Aufzeichnungen durch ihre stilistischen Überarbeitungen überhaupt erst den Charakter von Prophezeiungen und unabgeschlossenen ,Ahnungen'. Ihre Bearbeitungspraxis zielt auf eine Verbindung von inhaltlicher Geschlossenheit und formaler Offenheit, die für die romantische Poetik des Fragments charakteristisch ist. Während die Fragmente sich in Ton und Sprache „unanfechtbar“ und abgeschlossen, ohne argumentative „Herleitungen“ oder Relativierungen präsentieren, weisen sie formal „über sich selbst hinaus“: Sie stehen stets in einer Reihe mit anderen Fragmenten. ${ }^{40}$ Da ihr qualitativer Zusammenhang - also die Frage, ob sie in einem kausalen, gegensätzlichen oder ergänzenden Verhältnis zueinander stehen - nicht expliziert wird, bleibt es den Lesern überlassen, diese Leerstellen im Text zu füllen. Die Edition Tiecks und Schlegels zeugt von dem Bemühen, die Anforderungen an die Lektüre in dieser Hinsicht durch die künstliche Herstellung von Fragmenten und Textlücken noch zu steigern. Anders als in der Forschung konstatiert wurde, zielte ihre redaktionelle Tätigkeit also nicht darauf ab, Novalis "gefälliger“ und „lesbarer" zu machen. ${ }^{41}$ Die Lesbarkeit wird durch die Eingriffe vielmehr erheblich erschwert. Auf diese Weise erschaffen die Herausgeber den ersten ,Klassiker der Romantik', der mit seinen vermeintlichen „Ahndungen“ schier unerschöpfliche Auslegungspotenziale bietet.

\section{Unterbrochene Setzungen. Die Fragmentarisierung des Fragments}

Besonders anschaulich wird die Technik der Fragmentarisierung anhand jener Textauszüge, die Schlegel und Tieck dem Allgemeinen Brouillon entnommen haben. Das Allgemeine Brouillon ist eine nachgelassene Sammlung

39 Müller, „Vorlesungen über die deutsche Wissenschaft und Literatur“ (Anm. 7), S. 56.

40 So die Charakterisierung von Schlegels Fragmentpoetik bei Ansgar Maria Hoff, Das Poetische der Philosophie. Friedrich Schlegel, Friedrich Nietzsche, Martin Heidegger, Jacques Derrida, Alfter: Denkmal 2002, S. 51.

41 William Actander O'Brian, „Herstellung eines Mythos. Novalis' ,Schriften“ in der redaktionellen Bearbeitung von Tieck und Schlegel“, in: Zeitschrift für deutsche Philologie 111 (1992), H. 2, S. 161-18o, hier S. 162. 
von Aufzeichnungen aus den Jahren 1798 und 1799. Dabei handelt es sich um eine Art Lektüretagebuch, das allerdings keine textnahen Exzerpte, sondern eigene Anregungen und Gedanken enthält, die Hardenberg aus der Lektüre entwickelte. Die Funktion der Sammlung bestand darin, Materialien oder Vorstudien zu einem enzyklopädischen Projekt zu kompilieren, das die Verbindungen der unterschiedlichen wissenschaftlichen Fachgebiete darstellen sollte. ${ }^{42}$ Die Lesestoffe, die den Anlass für die Aufzeichnungen gaben, lassen sich oft nur ansatzweise erschließen, weil in der Regel lediglich indirekte Hinweise auf die Schriften, aber kaum Autorennamen oder Werktitel fallen. ${ }^{43}$ Schlegel und Tieck haben bei der Bearbeitung des Nachlasses einzelne Textstellen aus dieser Sammlung isoliert und fragmentarisiert. Sie machten nicht nur textuelle Bezüge und Paraphrasen fremder Gedanken unkenntlich, sondern unterbrachen auch die Kontinuität der Aufzeichnungen. Die „Fragmente" aus dem Allgemeinen Brouillon erhalten in den Schriften somit einen völlig veränderten Charakter und neue Kontexte. Was in Hardenbergs Notizen bisweilen noch als unausgegorene Überlegung oder abwägende Bemerkung erscheint, wird hier zur apodiktischen Setzung.

Ein Beispiel dafür bietet das 18. Fragment der Schriften, das aus einer längeren Aufzeichnung des Allgemeinen Brouillons stammt. Hardenberg befasst sich darin - im Anschluss an seine Lektüre von Fichtes Versuch einer neuen Darstellung der Wissenschaftslehre $(1797)^{44}$ - mit dem Zusammenhang von Philosophie und Charakter. Sein Urteil fällt in den beiden Fassungen unterschiedlich deutlich aus:

42 Vgl. Hans-Joachim Mähl, „Einleitung“, in: Friedrich von Hardenberg, Novalis Schriften. Historisch-kritische Ausgabe, Bd. 3, hrsg. von Richard Samuel in Zusammenarbeit mit Hans-Joachim Mähl und Gerhard Schulz, Stuttgart: Kohlhammer 1983, S. 207-241, hier bes. S. $237 \mathrm{ff}$.

43 Vgl. Hans-Joachim Mähl, „Probleme der quellenkritischen Kommentierung, behandelt an Textbeispielen der Novalis-Edition“, in: Louis Hay und Winfried Woesler (Hrsg.), Die Nachlassedition. La publication de manuscrits inédits, Bern u.a.: Lang 1979, S. 103-118, hier bes. S. $109 \mathrm{ff}$.

44 Vgl. Johann Gottlieb Fichte, „Versuch einer neuen Darstellung der Wissenschaftslehre“, in: Philosophisches Journal VII (1797), S. 1-20. 


\section{Allgemeines Brouillon}

Manche verändern ihre Phil[osophie] wie ihre Dienstbothen und Wünsche. Am Ende fangen sie das ganze Geschlecht an zu hassen und wählen zum letztenmal, aber auf immer. $\mathrm{Ob}$ sie sich dann gerade in der richtigen Stimmung eines Wählers befinden, will ich hiernicht entscheiden. Kurz sie glauben nun von der Phil[osophie] los zu seyn und sind mehr, als je in den Händen dieses Daemons, der sie nun gut füttert und pflegt, um sie zu einem schmackhaftern Bissen für sich zuzurichten. ${ }^{45}$
Fragmente vermischten Inhalts

Manche verändern ihre Philosophie wie ihre Dienstbothen und Wünsche. Am Ende hassen sie alle Art davon und wählen zum letztenmal, aber auf immer. Nun glauben sie von der Philosophie los zu seyn und sind nicht mehr, als je in den Händen dieses Daemons, der sie nun gut füttert und pflegt, um sie zu einem schmackhaften Bissen für sich zuzurichten. -46

Dass sich ein „ich“ "zu Wort meldet, das über eine Frage noch nicht abschließend „entscheiden“ will, ist in der romantischen Poetik des Fragments nicht vorgesehen. Tieck und Schlegel verleihen den Textauszügen durchweg einen generalisierenden und allgemeingültigen Charakter, der die Möglichkeit alternativer Sichtweisen oder relativierender Einwände ausschließt. Diese Poetik, die auch die authentischen Fragmente Hardenbergs charakterisiert, resultiert aus der philosophischen Einsicht, dass das Bewusstsein aus einer Aneinanderreihung bruchstückhafter Erlebnisse besteht. ${ }^{47}$ Wie Hardenberg vertrat auch Schlegel in seiner Auseinandersetzung mit Fichtes Subjektphilosophie die Ansicht, dass ganzheitliche Erfahrungen des eigenen Selbst wie auch der Welt unmöglich seien. Die Erkenntnis des sogenannten „Absoluten“ - der Einheit zwischen der Vorstellung und den Dingen - befinde sich vielmehr in einem permanenten Aufschub. Die Fragmente der Romantiker bringen dieses Problem in ambivalenter, ironischer Weise zum Ausdruck. Durch ihre apodiktische Form sind sie der "mißlungene[] Ausdruck des Absoluten [...], das als

45 Hardenberg, Novalis Schriften, Bd. 3 (Anm. 42), S. 338, Herv. E. T.

46 Friedrich von Hardenberg, Novalis Schriften, hrsg. von Friedrich Schlegel und Ludwig Tieck, Zweiter Theil, Berlin: Verlag der Realschulbuchhandlung 1802, S. $264 f$.

47 Dass auch Hardenberg selbst diese Fragmentpoetik vertrat, zeigt Jurij Striedter, Die Fragmente des Novalis als „Präfigurationen“ seiner Dichtung, München: Fink 1985, S. 33f. Vgl. dazu auch eine Bemerkung aus dem Allgemeinen Brouillon: „Man muß nicht ungewiß etc. ängstlich etc. schreiben - verworren, unendlich - sondern bestimmt - klar - fest - mit apodiktischen, stillschweigenden Voraussetzungen." (Hardenberg, Novalis Schriften, Bd. 3 [Anm. 42], S. 410) 
solches unfaßlich bleibt “. ${ }^{48}$ Sie nehmen Setzungen vor, die sie durch ihre offene Form und die Unterbrechung des Gedankens sogleich wieder relativieren.

Die Bearbeitungen von Novalis' Aufzeichnungen machen deutlich, dass dieses fragmentarische Bewusstsein des modernen Subjekts kein transzendentaler Zustand, sondern das Ergebnis textueller Strategien ist. Die Poetik des „zerrissenen Selbst ${ }^{449}$ muss das „Ich“ und seine abwägenden Reflexionen erst wieder aus dem Text streichen, um die Unzulänglichkeiten der Selbst- und Weltwahrnehmung treffend zum Ausdruck zu bringen. Was als Symbol eines konstitutiven Mangels erscheinen soll, muss diesen Mangel zuallererst schaffen. Das Fragment ist in den Schriften nicht sekundärer Ausdruck eines bruchstückhaften Subjekts, sondern dieses Subjekt ist umgekehrt der Effekt editorialer Kürzungen, die die Texte zu Bruchstücken machen. Im Fall der Nachlassedition von Novalis erzeugen die Paratexte hingegen den Eindruck, der Autor sei aufgrund seiner Weltabgewandtheit gar nicht in der Lage gewesen, längere Gedanken und zusammenhängende Reflexionen zu Papier zu bringen. Zum Charakter seiner Lektüretagebücher und Aufzeichnungen wie denen des Allgemeinen Brouillons passt dieses Bild nur bedingt.

Der von den Herausgebern angestrebten fragmentarischen Textform als Ausdruck des Absoluten entsprechen die biographischen Texte insofern nur in eingeschränkter Weise, als sie an vielen Stellen einen nachdenklichen Charakter haben und individuelle Züge tragen. So streut Hardenberg in seine Notizen regelmäßig persönliche Anmerkungen ein. Eine Aufzeichnung über das Märtyrertum aus dem Nachlass endet etwa mit dem Ausruf: „O! daß ich Märtyrersinn hätte?" 50 Dieser Wunsch, den Novalis am 9. Oktober 1800 auch in sein Tagebuch notierte, erschien den Herausgebern für ihre „Fragmente“ möglicherweise zu persönlich oder zu wenig verallgemeinerbar - in jedem Fall ließen sie ihn bei ihrer Bearbeitung der Passage weg. Die Streichung des Satzes ist charakteristisch für die Bearbeitungspraxis Tiecks und Schlegels: Äußerungen in der ersten Person Singular und biographische Eintragungen kommen in den Fragmenten vermischten Inhalts so gut wie nicht vor. ${ }^{51}$

48 Manfred Frank, „Allegorie, Witz, Fragment, Ironie. Friedrich Schlegel und die Idee des zerrissenen Selbst“, in: Willem van Reijen (Hrsg.), Allegorie und Melancholie, Frankfurt/ Main: Suhrkamp 1992, S. 124-146, hier S. 139.

49 Ebd., S. 124.

$5^{\circ} \quad$ Hardenberg, Novalis Schriften, Bd. 3 (Anm. 42), S. 687.

51 Eine Ausnahme bilden etwa Notizen zur Subjektphilosophie Fichtes, bei denen die Formulierungen in der ersten Person Singular allerdings keine biographischen Züge haben. Vgl. als einen solchen Fall etwa das 21. Fragment der Schriften: „Fichte's Ausführung seiner Idee ist wohl der beste Beweis des Idealismus. Was ich will, das kann ich. Bei dem Menschen ist kein Ding unmöglich.“ (Hardenberg, Novalis Schriften, Zweiter Theil [Anm. 46], S. 266) 
Formulierungen wie „ich wundere mich“, „daher würd ich sehr geneigt sein“ oder "sollt ich jetzt krank werden“, die sich in den nachgelassenen Notizen vielfach finden, wurden in der Regel gar nicht erst übernommen oder aber umgeschrieben. ${ }^{52}$

Gerade jene Textpassagen, die dabei helfen könnten, den von Tieck geforderten Zusammenhang zwischen "Schriften“ und „Leben“ herzustellen, sind also gezielt eliminiert worden. Die Herausgeber führen eine Trennlinie zwischen Werk und Biographie ein, nur um die „befreundeten“ Leser dann aufzufordern, diese Grenze zu überschreiten und die Manifestationen des individuellen "Geistes" im Text ausfindig zu machen. Die Geschichte eines Autors aus seinen Schriften zu lesen, setzt in der romantischen Ästhetik der Edition offenbar voraus, dass zuvor alle Hinweise auf diese Geschichte getilgt wurden. Damit das Publikum selbst hermeneutisch aktiv werden konnte, mussten die Herausgeber zunächst für ein biographisches Informationsdefizit sorgen. Das gilt nicht bloß für die Vorrede, sondern auch für die Fragmente selbst.

Hinzu kommt, dass die Notizen des Allgemeinen Brouillons an vielen Stellen argumentativ aufeinander aufbauen, also eine Kontinuität besitzen, die in den bearbeiteten „Fragmenten“ gezielt unterlaufen wird. In den redigierten Fassungen der Schriften fehlen in der Regel alle Signale, die fortlaufende Gedankengänge anzeigen - insbesondere Konjunktionen und Adverbien wie „also“, „oder“ und „daher“. ${ }^{53}$ Die „Fragmente“ bestehen aus Konstativsätzen, die jeweils separat für sich stehen. Bezüge zum Vorangegangenen sind darin nicht vorgesehen und werden von den Herausgebern häufig gekürzt. Eine Notiz mit dem Titel „Physiologie“ enthält etwa im Original den Satz: „Wir sahen vorhin, daß Bildung und Vermehrung der Seele das wichtigste und erste Unternehmen

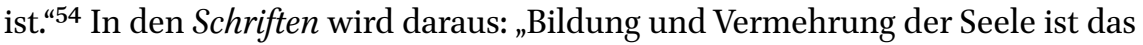
wichtigste und erste Unternehmen. “55 Die von Tieck und Schlegel redigierten Texte behaupten Sachverhalte, auf deren Begründung oder Herleitung sie bewusst verzichten.

Dasselbe gilt für die bildliche Rede. Figürliche Ausdrücke werden nicht in der Form von Vergleichen eingebracht, sondern beanspruchen den

52 Vgl. Hardenberg, Novalis Schriften, Bd. 3 (Anm. 42), S. 188, S. 437 und S. 473 . Mit dieser Vorgehensweise schlossen Schlegel und Tieck an Hardenbergs eigene Überarbeitung des Allgemeinen Brouillons an. Im Herbst 1798 begann er mit der Revision der Sammlung, klassifizierte die Notizen nach Sachgebieten und strich Biographisches sowie provisorische Notizen. Vgl. Mähl, „Einleitung“ (Anm. 42), S. 208.

53 Vgl. Hardenberg, Novalis Schriften, Bd. 3 (Anm. 42), S. 314, S. 385 und S. 471.

54 Ebd., S. 318.

55 Hardenberg, Novalis Schriften, Zweiter Theil (Anm. 46), S. 342. 
Stellenwert absoluter Metaphern. Schreibt Hardenberg etwa in einer Notiz, das „Denken“ sei „wie die Blüthe“, die die "feinste Evolution“ der "plastischen Kräfte" darstelle, ${ }^{56}$ so fehlt dieser Vergleich in der bearbeiteten Fassung der Schriften. Übrig bleibt nur noch der Folgesatz: „Die Denkorgane sind die Welterzeugungs-, die Naturgeschlechtstheile. “57 Das Blütengleichnis, das der Herleitung dieser Metapher diente, ist in der Version Schlegels und Tiecks eliminiert worden. Damit verliert die Analogie zwischen organologischen und kognitiven Prozessen scheinbar ihre Arbitrarität: Statt nur der Veranschaulichung zu dienen, erhält sie den Anschein eines substanziellen Zusammenhangs. Eine ähnliche Umschrift haben die Editoren mit der 775. Notiz des Allgemeinen Brouillons vorgenommen. Hier steht im Original: „Wir haben uns [...] einen Gott wie einen Monarchen gewählt." In den Schriften steht an dieser Stelle: „Wir haben uns [...] einen Gott zu einem Monarchen gewählt.“58 Die Metapher wird hier nicht nur verabsolutiert, sondern vor allem geht die Pointe des Satzes - die Idee, dass der transzendente Gott von den Menschen wie ein irdischer Herrscher gewählt wurde - in der Bearbeitung verloren. Die Voraussetzungen von Hardenbergs Thesen und Äußerungen sind in der publizierten Fassung der Fragmente nicht mehr sichtbar.

Die Syntax der Fragmente bleibt von der Poetik der Verknappung hingegen ausgenommen. Die Tendenz zur Beseitigung biographischer, erläuternder oder einschränkender Sätze und Formulierungen geht überraschenderweise auf der Ebene des einzelnen Satzes mit einer Vervollständigung von Lücken einher. Viele der Notizen Hardenbergs haben eine elliptische Form, in der die Verben oder Pronomina fehlen. In den Schriften sind diese Sätze durch Tieck und Schlegel in der Regel ergänzt und komplettiert worden: Die Bemerkung „Predigt ist - Bruchstück der Bibel“ wird etwa ergänzt zu dem Satz: „Eine Predigt ist ein Bruchstück der Bibel“ ${ }^{59}$ Aus der fragmentarischen Notiz „Die Zeit der allg Anarchie - Gesetzlosigkeit - Freyheit [...]“ wird die Aussage: „[H]ier tritt die Zeit der allgemeinen Anarchie, der Gesetzlosigkeit, Freiheit [...] ein“. ${ }^{60}$ Der Entwurf einer Aufzeichnung „Über die allg n [sic] Sprache der Musik“ wird in den Schriften zu einem Deklarativsatz: „Die Musik redet eine

\footnotetext{
$56 \quad$ Hardenberg, Novalis Schriften, Bd. 3 (Anm. 42), S. 476.

57 Hardenberg, Novalis Schriften, Zweiter Theil (Anm. 46), S. 325.

58 Hardenberg, Novalis Schriften, Bd. 3 (Anm. 42), S. 418; vgl. dazu Hardenberg, Novalis Schriften, Zweiter Theil (Anm. 46), S. 473 f.

59 Hardenberg, Novalis Schriften, Bd. 3 (Anm. 42), S. 435; vgl. Hardenberg, Novalis Schriften, Zweiter Theil (Anm. 46), S. 49o.

6o Hardenberg, Novalis Schriften, Bd. 3 (Anm. 42), S. 28o; vgl. Hardenberg, Novalis Schriften, Zweiter Theil (Anm. 46), S. $518 f$.
} 
allgemeine Sprache". ${ }^{61}$ Wo ein Satz noch keine thetische Form hat, erhält er sie durch die Redaktoren. Dabei werden auch grammatische Verstöße oder missverständliche Formulierungen für den Druck berichtigt. ${ }^{62}$

Typographisch erwecken Novalis' Fragmente vermischen Inhalts in der Ausgabe Schlegels und Tiecks den Eindruck eines langen, unterbrochenen Prosatexts. Ungeachtet der geschlossenen, deklarativen Form jedes einzelnen Fragments suggerieren Interpunktion und typographische Darstellung einen übergreifenden Zusammenhang zwischen den Textteilen. Die Fragmente sind, anders als in der von Richard Samuel herausgegebenen kritischen Ausgabe, nicht nummeriert und nicht durch Leerzeilen separiert. Dies entsprach dem ausdrücklichen Wunsch Tiecks, der den Verleger Reimer Anfang Oktober 1802 bat, die „verschiedenen Fragmente nur durch einen -- und eine Einschiebung“ graphisch voneinander abzugrenzen. ${ }^{63}$ Innerhalb dieser Abschnitte tauschte er hingegen fast alle Gedankenstriche und teilweise sogar die Punkte gegen Kommata aus. Dadurch entsteht der Eindruck eines lückenlosen Gedankenflusses, wie die Bearbeitung einer weiteren Notiz aus dem Allgemeinen Brouillon für die Schriften besonders gut zeigt:

Allgemeines Brouillon

Die Philosophie ist von Grund auf antihistorisch. Sie geht vom Zukünftigen, und Nothwendigen nach dem Wircklichen - Sie ist die Wissenschaft des allgemeinen Divinations-Sinns. Sie erklärt die Vergangenheit aus der Zukunft, welches bey der Geschichte umgekehrt der Fall ist.

(Sie betrachtet isolirt, im Naturstande - unverbunden.) ${ }^{64}$

\section{Fragmente vermischen Inhalts}

Die Philosophie ist von Grund aus antihistorisch, sie geht vom Zukünftigen und Nothwendigen nach dem Wirklichen, sie ist die Wissenschaft des allgemeinen Divinations-Sinns, sie erklärt die Vergangenheit aus der Zukunft, welches bei der Geschichte umgekehrt der Fall ist. ${ }^{65}$

61 Hardenberg, Novalis Schriften, Bd. 3 (Anm. 42), S. 283; vgl. Hardenberg, Novalis Schriften, Zweiter Theil (Anm. 46), S. 359.

62 Vgl. etwa die Notiz „Die Welt des Märchens ist die durchausentgegengesetzte Welt der Welt der Wahrheit", die in den Schriften folgendermaßen umgeschrieben wird: „Die Welt des Mährchens ist die der Welt der Wahrheit durchaus entgegen gesetzte, und eben darum ihr so durchaus ähnlich“ (Hardenberg, Novalis Schriften, Bd. 3 [Anm. 42], S. 435; vgl. Hardenberg, Novalis Schriften, Zweiter Theil [Anm. 46], S. 519).

63 Brief von Tieck an Georg Andreas Reimer im Oktober 1802, in: Ludwig Tieck, Letters of Ludwig Tieck. Hitherto unpublished 1792-1853, hrsg. von Edwin H. Zeydel, Percy Matenko and Robert Fyfe, New York: MLA 1937, S. 5 o.

64 Hardenberg, Novalis Schriften, Bd. 3 (Anm. 42), S. $464 f$.

65 Hardenberg, Novalis Schriften, Zweiter Theil, (Anm. 46), S. 167. 
Durch die konsequente Einfügung von Kommata wird die parataktische Struktur der Aufzeichnung zu einer stetigen Abfolge von Setzungen. Die „Fragmente" erwecken den Anschein einer textuellen Ganzheit, die immer wieder unterbrochen wird, aber nie gänzlich abbricht. Sie sollen offenbar nicht wie aphoristische Sinnsprüche gelesen werden, die aus ihrer textuellen Umgebung ohne Verluste isoliert werden können und für sich stehen, sondern - ganz im Sinne von Tiecks Vorwort - als Teile eines Ganzen, dessen Leerstellen gefüllt und dessen fehlende Stücke rekonstruiert werden müssen. Erst durch die graphische Markierung der Lücken zwischen den Fragmenten mithilfe von Gedankenstrichen wird sowohl deren Bruchstückhaftigkeit als auch das Ganze selbst sichtbar gemacht.

Damit wird deutlich, dass Fragmentästhetik und Ganzheitsästhetik mehr gemeinsam haben, als man auf den ersten Blick meinen könnte. Wenn Goethe seine Schriften im siebten Buch von Dichtung und Wahrheit 1808 als „Bruchstücke einer großen Konfession“ bezeichnete, ${ }^{66}$ dann beweist dies, dass ganze Werke um 1800 ebenso als Fragmente gelesen werden konnten wie Fragmente als Ausdruck einer Ganzheit interpretierbar waren ${ }^{67}$ In beiden Fällen war die Kongenialität des Auslegers gefragt, der die Individualität des Verfassers aus dessen Schriften lesen musste. Auch Schlegel betonte in seiner Rezension von Goethes Wilhelm Meister, dass dieser Roman die "gewöhnlichen Erwartungen von Einheit und Zusammenhang“ zwar nicht erfülle. Doch wer „echten systematischen Instinkt" und „Sinn für das Universum“ habe, „fühlt gleichsam überall die Persönlichkeit und lebendige Individualität des Werks, und je tiefer er forscht, je mehr innere Beziehungen und Verwandtschaften, je mehr geistigen Zusammenhang entdeckt er in demselben.“68 Das ideale Werk, so scheint es, ist immer zugleich fragmentarisch und ganz: Als Geschriebenes ist es konstitutiv lückenhaft und unvollständig; als Ausdruck eines Geistes, der im Akt der Lektüre verstanden und erfasst werden kann, ist es dagegen eine integrale, ganzheitliche Einheit.

66 Johann Wolfgang Goethe, Aus meinem Leben. Dichtung und Wahrheit, hrsg. von KlausDetlef Müller, Frankfurt/Main: Deutscher Klassiker Verlag 2007, S. 310.

67 Auch in seiner Winckelmann-Biographie verbindet Goethe Fragment- und Ganzheitsästhetik, vgl. Johann Wolfgang Goethe, Winckelmann und sein Jahrhundert, in Briefen und Aufsätzen, hrsg. von Goethe, Tübingen: Cotta 1805. Vgl. auch Daniel Ehrmann, „Fragmentierung und Nekromantie. Strategien und Mechanismen der Legitimation biographischer Darstellung um 1800“, in: Christian Klein und Falko Schnicke (Hrsg.), Legitimationsmechanismen des Biographischen, Bern u.a.: Lang 2016, S. 57-9o, bes. S. 73ff.

68 Friedrich Schlegel, „Über Goethes Meister“, in: Schlegel, Kritische Friedrich-SchlegelAusgabe, Bd. 2 (Anm. 31), S. 126-147, hier S. 133. 


\section{Epochenpolitik. Der Klassiker der Romantik}

Während Goethe mit seiner Autobiographie die Deutung seiner bruchstückhaften „Konfession“ gezielt steuerte, war die Lage bei Novalis durch die zurückhaltenden biographischen Angaben der Herausgeber etwas unklarer. Bei den Lesern des 19. Jahrhunderts hat die Frage, was der Zusammenhang des „Ganzen“, das Schlegel und Tieck den „Geist“ des Autors nannten, eigentlich sei, hermeneutische Energien in hohem Maß freigesetzt. Das Publikum der Schriften ist so gesehen Teil jenes Kollektivs, das den Autor Hardenberg hervorgebracht und sein Bild für lange Zeit geprägt hat. Weitgehender Konsens herrschte bei den Lesern darüber, dass der "göttliche Jüngling“ Novalis, der „nur auf der Erde wandelte, um sich bald wieder zu dem geliebten Lande seiner Sehnsucht aufzuschwingen“, nichts als „Andeutungen“ seiner eigentlichen Ideen hinterlassen habe. Was er "gewollt" habe, könne man nur aus seinen „Fragmenten“ erahnen. ${ }^{69}$ Unabhängig davon, ob diese Fragmentarizität als Verlust oder als besondere Qualität gewertet wurde, war sie für die Zeitgenossen der Inbegriff eines "rein“ poetischen Daseins. Für Joseph Görres etwa besaß Novalis ein „Gemüth“, das so sehr „in sich selbst verschlossen“, "contemplativ" und in die Anschauung "verloren“ war, dass die Ideen aus seiner "dunkeln Tiefe“ nicht vollständig zu Tage treten konnten. ${ }^{70}$ Und der französische Literaturkritiker Henri Blaze war 1839 sogar überzeugt davon, dass Novalis' Werke, hätte er sie vollenden können, ,jetzt vergessen“ wären, weil der „Lebenshauch“ und die „wundervollen Einzelheiten“ seiner Poesie „in einer allzu großartigen Anlage zerstreut" worden wären. ${ }^{71}$

Die Aufgabe, die Tieck in seiner Vorrede 1802 formuliert hatte - die innere Geschichte Hardenbergs aus seinem Werk zu erkennen -, lösten die Leser des 19. Jahrhunderts also, indem sie die Form der Texte selbst zum Schlüssel der Deutung machten. Die fragmentarische Gestalt der Schriften wurde zum Spiegel der Persönlichkeit des Autors. Die ,Lücken` im Text, die - zumindest was die Fragmente betraf - auf die planmäßigen Eingriffe der Herausgeber zurückgingen, wurden auf die Weltabgewandtheit eines in Geist und Poesie versunkenen Mystikers zurückgeführt. Für die Klarheit zusammenhängender Prosa schien Hardenberg schlicht nicht geschaffen gewesen zu sein. Dass die

69 Anonymus, „Hardenberg“, in: Conversations-Lexicon oder encyclopädisches Handwörterbuch für gebildete Stände, Bd. 4, Leipzig, Altenburg: Brockhaus 1815, S. 536-537, hier S. 537.

70 Joseph Görres, „Mystik und Novalis“, in: Aurora, eine Zeitschrift aus dem südlichen Deutschland, Mittwoch, den 3. April; Freitag, den 5. April 1805, Nr. 4O-41, S. 157f. und S. 161f., hier S. 162 .

71 Henri Blaze, „Ueber Goethe's Faust“, in: A. Lewald's Europa. Chronik der gebildeten Welt 5 (1839), H. 3, S. 57-68, hier S. 61. 
Schriften mit den längeren Romanfragmenten (Heinrich von Ofterdingen, Die Lehrlinge von Sais) begannen und nach den lyrischen Dichtungen in immer kleiner werdenden Texteinheiten und Fragmenten endeten, konnte - trotz der fehlenden Chronologie - als Indiz für die zunehmende Lebensferne und Entrücktheit des Autors gedeutet werden.

Die dunklen Stellen des Werks wurden zum Faszinosum und zugleich zum Beweis für dessen poetische Qualität. Die Schriften entsprachen damit jenen Kriterien, die Schlegel bereits im Jahr 1800 als das Merkmal "classischer" Texte bestimmt hatte: Wie die antiken oder mittelalterlichen Klassiker war auch Novalis so schwer zu verstehen, dass er wiederholt und "cyclisch“ gelesen werden musste, ohne dabei je "ganz" erfasst zu werden..$^{72}$ Als „rätselvolle[r] seher" und als "seelisch tiefster" Romantiker wurde er bald zu einem Lieblingsautor der deutschen Philologie. ${ }^{73}$ Dieses Bild hat sich in der Germanistik bis in die Mitte des 2o. Jahrhunderts erhalten, die an den Schriften Hardenbergs immer wieder die "helle Spur unendlichen Sinns“ und die unendlichen Potenziale der Deutung bewunderte. ${ }^{74}$

Dennoch waren Hardenbergs „Ahndungen“ nicht völlig beliebig auslegbar. Auch nach mehrfacher Lektüre und nach mehreren Jahrzehnten der NovalisRezeption blieben bestimmte Äußerungen stärker im Bewusstsein der Leserschaft verankert als andere. Auch dafür waren die redaktionellen Eingriffe der Herausgeber mit verantwortlich. Ihnen ging es nicht nur um ein spezifisches, hermeneutische Ansprüche herausforderndes Werk- und Autorenbild, sondern auch um eine literaturgeschichtliche Selbstpositionierung. Diese kulturpolitischen Ambitionen, die sich im Laufe der Zeit immer wieder verschoben und die Textauswahl und -anordnung der Auflagen veränderten, ${ }^{75}$ unterwandern das Bild von Novalis als einem der Gegenwart entrückten Klassiker der romantischen Ära. Um die Schriften in gegenwärtigen Kontroversen zum

72 Friedrich Schlegel, „Über die Unverständlichkeit“, in: Schlegel, Kritische Friedrich-SchlegelAusgabe, Bd. 2 (Anm. 31), S. 363-372. Vgl. zu diesem aus Leserperspektive konzipierten Begriff des Klassischen auch Nikolaus Wegmann, „Was heißt einen ,klassischen Text lesen? Philologische Selbstreflexion zwischen Wissenschaft und Bildung“, in: Jürgen Fohrmann und Wilhelm Voßkamp (Hrsg.), Wissenschaftsgeschichte der Germanistik im 19. Jahrhundert, Stuttgart, Weimar: Metzler 1994, S. 334-45o.

73 Oskar Walzel, „Meissner und Wille, Novalis sämtliche Werke“, in: Anzeiger für deutsches Altertum (1900), S. 237-250, hier S. 237.

74 Edgar Hederer, Novalis, Wien: Amandus 1949, S. 7.

75 Einen Überblick bietet z.B. Sophia Vietor, „Die Erstdrucke und die Erstausgaben der Schriften von Novalis und ihre Herausgeber", in: Gabriele Rommel (Hrsg.), Novalis. Das Werk und seine Editoren, Wiederstedt: Novalis Forschungs-Stätte für Frühromantik und Novalis-Museum 2001, S. 65-85, hier S. $72 \mathrm{ff}$. 
Einsatz zu bringen, sorgten die Herausgeber in einigen Themenbereichen für polemische Zuspitzungen und vereinseitigten die Äußerungen ,ihres' Autors.

Bereits das Phantasma einer literarischen Heilsgeschichte, die angeblich von Lessing zu Novalis verlief, machte dieses Interesse an einer Profilierung der eigenen Epoche deutlich. Novalis' Schriften wurden von den Herausgebern dazu genutzt, nicht nur eine „Erneuerung der Künste“ zu verkünden, sondern zugleich eine Abgrenzung von den etablierten Künsten zu markieren. ${ }^{76}$ Eine der zentralen Rezeptionslinien, die Tieck und Schlegel in diesem Zusammenhang prägten, war die vermeintliche Gegnerschaft von Goethe und Novalis. ${ }^{77}$ In der Rede von der "Goethe-Klassik“ und der „Novalis-Romantik“ konnte sich diese Antithese langfristig halten. ${ }^{78}$ Goethe war für Schlegel wie für Tieck im Entstehungszeitraum der Novalis-Ausgabe gleichermaßen mit Vorbildhaftigkeit und Einflussangst assoziiert. ${ }^{79}$ Tieck war vor allem daran interessiert, seinen Bildungsroman Franz Sternbalds Wanderungen (1798) als romantische Eigenleistung zu rechtfertigen und als eigentliches Vorbild für Hardenbergs Heinrich von Ofterdingen darzustellen. ${ }^{80}$

Schlegel wiederum unternahm trotz seiner offenkundigen GoetheVerehrung immer wieder Versuche, Goethes Dichtungen als eine Vorstufe der romantischen Ära darzustellen. Die Novalis-Edition befindet sich im Zwischenzeitraum einer Phase der bedingungslosen Goethe-Verehrung Schlegels und einer folgenden Phase, in der er sich überwiegend kritisch zu Goethe äußerte. ${ }^{81}$ Während private Studienhefte Schlegels bereits zu Beginn des 19. Jahrhunderts negative Bemerkungen enthalten, lässt sich in den publizierten Aussagen dieser Zeit allenfalls eine leichte Relativierung der Goethe-Verehrung beobachten: Hatte Schlegel den Wilhelm Meister 1798 im Athenäum neben

$7^{6}$ Den Beginn einer „neue[n] Epoche der deutschen Literatur“ verkündet Schlegel - u.a. ebenfalls mit Bezug auf Novalis - auch in seiner Rezension von Adam Müllers literaturgeschichtlichen Vorlesungen, vgl. Friedrich Schlegel, „Vorlesungen über die deutsche Wissenschaft und Literatur von Adam H. Müller", in: ders., Kritische Friedrich-SchlegelAusgabe, Bd. 3, hrsg. von Hans Eichner, Paderborn u.a.: Schöningh 1975, S. 145-158, hier S. 156 .

77 Vgl. zum Folgenden den umfassenden und mit zahlreichen Belegen versehenen Aufsatz von Hans-Joachim Mähl, „Goethes Urteil über Novalis“, in: Jahrbuch des freien deutschen Hochstifts (1967), S. 130-270.

78 Franz Schultz, Klassik und Romantik der Deutschen, Bd. II, Stuttgart: Metzler 1952, S. 251f.

79 Zum Begriff der Einflussangst, der das Ringen von Autoren um eine „Anfangschance“ in der Auseinandersetzung mit einem „starken Vorläufer“ bezeichnet, vgl. Harold Bloom, Einfluss-Angst. Eine Theorie der Dichtung, Frankfurt/Main: Stroemfeld 1973, bes. S. 9 und 12.

8o Vgl. Vietor, „Die Erstdrucke“ (Anm. 75), S. 74.

81 Vgl. Robert Mandelkow, Goethe in Deutschland. Rezeptionsgeschichte eines Klassikers, Bd. 1, München: Beck 1980, S. 5off. 
Fichtes Wissenschaftslehre und der Französischen Revolution als eine der "größten Tendenzen" des Zeitalters gewürdigt, ${ }^{82}$ so legte er im Jahr 1800 Wert darauf, dass der Begriff der "Tendenz" nicht nur eine Würdigung, sondern auch ein Defizit oder eine Unvollkommenheit zum Ausdruck bringe. Damit warf er zugleich die Frage auf, wer diese „Tendenzen“ zur Vollendung bringen könne:

Ob ich nun der Meinung sei, [...] diese Tendenzen würden durch mich selbst in Richtigkeit und zum Beschluß gebracht werden, oder vielleicht durch meinen Bruder oder durch Tieck [...] oder erst durch einen Sohn von uns, durch einen Enkel, einen Urenkel [...] oder niemals; das bleibt der Weisheit des Lesers [...] anheim gestellt. ${ }^{83}$

Obwohl Schlegel die Position eines konkreten Nachfolgers offen lässt, bringt er mit der Rede von der unabgeschlossenen „Tendenz“ die Möglichkeit einer Überbietung Goethes oder Fichtes überhaupt erst ins Spiel.

Zwar musste auch Novalis' Werk in der Darstellung Tiecks und Schlegels „Tendenz" bleiben. Doch das Tendenzhafte seiner Dichtung scheint für die Herausgeber immerhin eine Steigerung der Goethe'schen Tendenzen darzustellen. Sie machen die Schriften zum Vehikel einer Goethe-Kritik, die bis dahin keiner von ihnen selbst so offensiv formuliert hatte. ${ }^{84}$ Tieck und Schlegel wählten aus der „Fülle von Goethe-Aufzeichnungen“, die Novalis notierte, nur wenige Fragmente aus, die sie teilweise stark manipulierten und umstellten:

\section{[Aufzeichnungen über Goethe]}

Gegen Wilhelm Meisters Lehrjahre. Es ist im Grunde ein fatales und albernes Buch - so pretentiös und pretiös - undichterisch im höchsten Grade, was den Geist betrift - so poëtisch auch die Darstellung ist. Es ist eine Satyre auf die Poësie, Religion etc. ${ }^{85}$

\section{Fragmente vermischten Inhalts}

Wilhelm Meister ist eigentlich ein Candide, gegen die Poesie gerichtet; das Buch ist undichterisch in einem hohen Grade, was den Geist betrifft, so poetisch auch die Darstellung ist. $^{86}$

82 Friedrich Schlegel, „Über Goethes Meister“ (Anm. 68), S. 198.

83 Schlegel: „Ueber die Unverständlichkeit“ (Anm. 72), S. 367.

84 Schlegel war, was seine eigene Goethe-Kritik anging, sehr vorsichtig, und hoffte etwa, dass Goethe die Ironie in seinem Fragment über die Tendenzen nicht bemerken würde. Erst später formulierte er seine Kritik direkt. Vgl. Hendrik Birus, „Größte Tendenz des Zeitalters oder ein Candide gegen die Poesie gerichtet? Friedrich Schlegels und Novalis' Kritik des Wilhelm Meister" (22.01.2004), in: Goethezeitportal, unter http://www.goethezeitportal.de/db/wiss/goethe/meisterslehrjahre_birus.pdf (letzter Zugriff: 30.07.2017).

85 Hardenberg, Novalis Schriften, Bd. 3 (Anm. 42), S. $646 f$.

86 Hardenberg, Novalis Schriften, Zweiter Theil (Anm. 46), S. 371. 
Zwar sind bereits Hardenbergs eigene Entwürfe in einem scharfen Ton verfasst - doch es handelte sich dabei vor allem um vorläufige Lektürenotizen, die von ebenso wohlwollenden Urteilen begleitet wurden. Tieck und Schlegel wählten die kritischen Passagen gezielt aus, schrieben sie zu pointierten Verdikten um und machten sie öffentlich. ${ }^{87}$ Ein Effekt dieser Maßnahmen besteht darin, dass Novalis' eigener Bildungsroman als poetischer Gegenentwurf zu dem "undichterisch[en]“ und prosaischen "Evangelium der Oeconomie“ Goethes erscheint. ${ }^{88}$ Die zahlreichen positiven Urteile Hardenbergs über Goethes Wilhelm Meister oder über Goethe selbst kommen in den Fragmenten vermischten Inhalts fast nicht vor. Auch wenn Tieck später versuchte, das Bild vom Goethe-Kritiker Novalis wieder zu korrigieren, ${ }^{89}$ blieb dieser dem Publikum als vehementer Gegner des Weimarer Klassikers im Gedächtnis.

Selbst der begeisterte Goethe-Anhänger Varnhagen von Ense konstatierte 1805 unter dem Eindruck seiner Novalis-Lektüre - immerhin 27 Jahre vor Goethes Tod -, dass „Goethe's schöne Zeit [...] verronnen“ sei. „Seine Philistrosität, die der prophetische Novalis durch die verschlungenen Gewebe des ,Wilhelm Meister' deutlich erkannt hatte, kommt immer mehr zu Tage“.90 In einer etwas anderen Weise griff auch Adam Müller den Gegensatz zwischen Goethe und Novalis auf. Demnach „ahndete" Novalis „den einzigen Vorwurf der gegen Goethe erhoben werden“ konnte: Er habe die „Allgegenwart des Christentums in der Geschichte und in allen Formen der Poesie und Philosophie" verkannt. ${ }^{91}$ Damit wurde Hardenberg zu einer Figur stilisiert, die den Übergang von Ökonomie in Poesie und von bürgerlicher Profanität in christliche Spiritualität ankündigte. ${ }^{92}$

Derartige Manipulationen und der allgemein sehr selektive Umgang mit dem Hardenberg-Nachlass ${ }^{93}$ waren der Grund dafür, dass die Edition Schlegels

87 Für einen ausführlichen Abgleich der ursprünglichen Notizen mit den Fragmenten Schlegels und Tiecks vgl. Mähl, „Goethes Urteil über Novalis“ (Anm. 77), S. $177 \mathrm{ff}$.

88 Hardenberg, Novalis Schriften, Zweiter Theil (Anm. 46), S. 371 .

89 Vgl. Mähl, „Goethes Urteil über Novalis“ (Anm. 77), S. 263.

$90 \quad$ Varnhagen, Aus dem Nachlaß Varnhagen's von Ense (Anm. 6), S. 239.

91 Müller, „Vorlesungen über die deutsche Wissenschaft und Literatur" (Anm. 7), S. 56 f.

92 Diese christliche Lesart von Novalis' Schriften unterstützten auch Schlegel und Karl von Hardenberg, vgl. dazu insbesondere die Publikationsgeschichte des Aufsatzes Die Christenheit oder Europa bei Vietor, „Die Erstdrucke“ (Anm. 75), S. 77ff.

93 Ähnlich selektiv gingen Tieck und Schlegel mit Hardenbergs Äußerungen zu Fichte um allerdings auf genau entgegengesetzte Weise. Hier wurde auf kritische Passagen gezielt verzichtet, sodass in den Schriften ausschließlich positive Würdigungen der Subjektphilosophie zu finden waren. Möglicherweise waren die engen persönlichen Beziehungen zu Fichte der Grund für diese Eingriffe, oder aber Schlegel beabsichtigte, das, was an Fichte „Tendenz" geblieben war, selbst mitteilen zu können (in seinen Kölner Vorlesungen 
und Tiecks seit dem späten 19. Jahrhundert, als sich die Universitätsphilologie für Novalis zu interessieren begann, als dilettantisch, verfälschend und unphilologisch galt. ${ }^{94}$ In der Wissenschaftsgeschichte gelten Tieck und Schlegel als ,Übergangsfiguren', die sich zwar für die Wiederentdeckung vergessener oder vernachlässigter Autoren eingesetzt haben, aber dennoch einer ,überkommenen', poetisierenden Editionspraxis verpflichtet blieben. Dies gilt insbesondere für die rege Herausgebertätigkeit Tiecks, der vor ästhetischen Korrekturen und verfälschenden Eingriffen in keinem seiner Editionsprojekte zurückschreckte. $^{95}$ Romantische und wissenschaftliche Editionstätigkeit erscheinen vor diesem Hintergrund als entgegengesetzte Paradigmen, die in einem zeitlichen Ablösungsverhältnis stehen.

Allerdings ist bemerkenswert, dass die romantische Philologie sich keineswegs an einem Massenpublikum orientierte, ${ }^{96}$ sondern mitunter dieselbe Leserschaft adressierte wie die wissenschaftliche Expertenphilologie. Wie sich gezeigt hat, ist die Edition Tiecks und Schlegels geradezu das Produkt einer neuen Aufmerksamkeit für philologisch geschulte Rezipienten. ${ }^{97}$ Sie wurde zunächst offenbar nicht für ein größeres Laienpublikum, sondern für eine kleinere Zahl interessierter und hermeneutisch versierter Leser konzipiert. Möglicherweise ist gerade diese Affinität $\mathrm{zu}$,modernen', philologischen Lektüreformen ein Grund dafür, dass sich die Universitätsphilologie schon bald vehement bemühte, Editoren wie Tieck als Dilettanten aus ihren Reihen auszusondern.

1804/o5), zumindest sollte es nicht von Novalis vorab artikuliert werden. Vgl. zu der einseitigen Auswahl der Fichte-Studien z.B. Hardenberg, Novalis Schriften, Bd. 3 (Anm. 42), S. 445, Nr. 924, und Hardenberg, Novalis Schriften, Zweiter Theil (Anm. 46), S. 273, wo u.a. der Satz fehlt: „Fichtes und Kants Methode ist noch nicht vollständig und genau genug dargestellt. [...] Alles ist so steif, so ängstlich noch." Zum Verhältnis von Schlegel und Fichte vgl. Bärbel Frischmann, Vom transzendentalen zum frühromantischen Idealismus, Paderborn u.a.: Schöningh 2005, S. 10gff.

94 Vgl. Walzel, „Meissner und Wille“ (Anm. 73), S. 239.

95 Vgl. z.B. Antoine Magen, „Der Philologe“, in: Claudia Stockinger und Stefan Scherer (Hrsg.), Tieck. Leben -Werk - Wirkung, Berlin, Boston: de Gruyter 2016, S. 424-440, hier S. 425f.; Rüdiger Krohn: „,... daß Alles Allen verständlich sey .... Die Altgermanistik und ihre Wege in die Öffentlichkeit“, in: Fohrmann/Voßkamp (Hrsg.), Wissenschaftsgeschichte (Anm. 72), S. 264-333, hier S. 285 ff.

96 Vgl. ebd., S. 285.

97 Ulrich Wyss spricht daher auch eher von unterschiedlichen Ausrichtungen oder Formen der Philologie um 180o, die das Spektrum des Fachs widerspiegeln. Vgl. Ulrich Wyss, „Der doppelte Ursprung der Literaturwissenschaft nach 1800“, in: Jürgen Fohrmann und Wilhelm Voßkamp (Hrsg.), Wissenschaft und Nation. Studien zur Entstehungsgeschichte der deutschen Literaturwissenschaft, München: Fink 1991, S. 73-88, hier S. 74ff. 
Die deutsche Philologie bildete im 19. Jahrhundert ein Ethos aus, das bewusste Revisionen und Manipulationen von Texten untersagte. Doch das Bemühen, die Klassizität der neueren deutschen Literatur durch editionsphilologische Strategien zu erzeugen und damit populäre oder politische Rezeptionen zu erschweren, teilte sie mit Tieck und Schlegel ebenso wie die Faszination für fragmentarische, unvollendete oder bruchstückhaft überlieferte Werke, die eines Editors und zahlreicher Ausleger bedürfen, um lesbar zu werden. Die Lücken im Text durften nicht mehr eigenmächtig erzeugt werden - aber sie zu deuten, den "Geist" des Ganzen zu bestimmen und ihren Zusammenhang durch die richtige Form der Anordnung zu erklären, blieb auch im Zeitalter der ,strengen` Wissenschaft ein Begehren der Editoren. 